\title{
Surgical Management of Isolated Level III Lymph Nodes in Breast Cancer
}

\author{
Chan Sub Park, M.D., Hyung-Kee Kim, M.D., Ho Yong Park, M.D., Ph.D. \\ Department of Surgery, School of Medicine, Kyungpook National University, Daegu, Korea
}

Purpose: The incidence of isolated level III lymph node recurrence in breast cancer was evaluated, and a surgical approach with preoperative tattooing was introduced. When a suspicious infraclavicular lymph node was detected, fine-needle aspiration cytology was performed to confirm the recurrence of breast cancer. In addition, surgical excision was considered to evaluate the biological characteristics of the recurrent lesion. Methods: The treatment strategy was determined through multidisciplinary team discussions, which included breast surgeons, plastic surgeons, oncologists, radiologists, pathologists, and radiation oncologists. Preoperative tattooing was performed, and the lymph nodes were removed after splitting the pectoralis major muscle. Results: Among 85 cases of locoregional recurrence, isolated recurrent lymph nodes were observed in 25 cases. Three patients with an isolated recurrent infraclavicular lymph node received radiotherapy, and six patients underwent excision biopsy due to a history of radiotherapy for primary tumors. There was no failure in identifying the recurrent lymph node and no major complication. Conclusion: The removal of isolated level III lymph node is safe, easy and can provide useful information for systemic therapy.

Key Words: Breast neoplasms, Lymph nodes, Recurrence

\section{INTRODUCTION}

The mortality rate of breast cancer has been decreasing for several decades $[1,2]$. Despite the improvement in survival rates, incidences of recurrence and metastasis are relatively higher for breast cancer than for other malignancies.

Breast cancer treatment strategies, including surgery, chemotherapy, radiotherapy, targeted therapy, and hormone treatment, are generally determined on the basis of tumor characteristics and should be offered as a personalized, tailored treatment. Therefore, tissue confirmation is necessary to evaluate biological characteristics even for a recurrent lesion.

Correspondence: Ho Yong Park, M.D., Ph.D.

Department of Surgery, School of Medicine, Kyungpook National University, 807 Hoguk-ro, Buk-gu, Daegu 41404, Korea

Tel: +82-53-200-2702, Fax: +82-53-200-2028, E-mail: phy123@knu.ac.kr

This research was supported by National Research Foundation of Korea (NRF) grants funded by the Korean government (2014R1A5A2009242, 2017R1C1B5076186, 2019R1A2C1006264) and by a grant from the National R\&D Program for Cancer Control, Ministry of Health and Welfare, Republic of Korea (1420040). In addition, this research was supported by a grant of the Korea Health Technology R\&D Project through the Korea Health Industry Development Institute (KHIDI), funded by the Ministry of Health \& Welfare, Republic of Korea (HI17C1142).

Received: Aug 21, 2019 Revised: Sep 29, 2019 Accepted: Feb 22, 2020
Thus far, there is no international consensus on the treatment strategy for isolated infraclavicular or interpectoral level III lymph node recurrence. Radiotherapy with chemotherapy may be considered preferentially. However, if a patient has already received radiotherapy or histologic confirmation is needed due to the failure of needle biopsy, excision of the recurrent lymph node should be considered. In addition, removal of the recurrent lesion may reduce the tumor burden, which may increase the therapeutic effect of palliative treatment [3].

Herein, the incidence of isolated level III lymph node recurrence in breast cancer was evaluated, and a surgical approach with preoperative tattooing was proposed.

\section{METHODS}

The clinical records and imaging findings of 1,390 patients with operable breast cancer were reviewed. All of the patients underwent surgery in Kyungpook National University Hospital from 1995 to 2015. The treatment strategy was determined through multidisciplinary team discussions, which included breast surgeons, plastic surgeons, oncologists, radiologists, pathologists, and radiation oncologists. After completion of the treatment for breast cancer, all the patients were followed for at least five years (biannually for the first two years and an- 
nually for the next three years) with blood tests, tumor marker tests, mammography, breast and neck ultrasonography, chest radiography, bone scan, chest/abdominal CT, or ${ }^{18 \mathrm{~F}}$ FDG PET/CT. The evaluated oncologic events were locoregional recurrence (including the ipsilateral breast and axillary, supraclavicular, and infraclavicular lymph nodes), distant metastasis, and death.

When a suspicious lymph node at level III was detected on chest CT, PET/CT, or neck ultrasonography, fine-needle aspiration cytology or core-needle biopsy was performed to determine post-procedural complications (Figure 1A-C). As a tissue sample is needed to evalu- ate biological characteristics, a needle biopsy was first considered. However, fine-needle aspiration cytology was performed when needle biopsy could not be performed due to the presence of important structures in the vicinity, and surgical excision was considered when metastasis was confirmed. After tumor characteristics were confirmed by needle biopsy or excision biopsy, additional chemotherapy or radiotherapy was administered.

All study participants provided informed consent, and the study design was approved by the appropriate ethics review board (IRB No. KNUCH 2015-05-205).
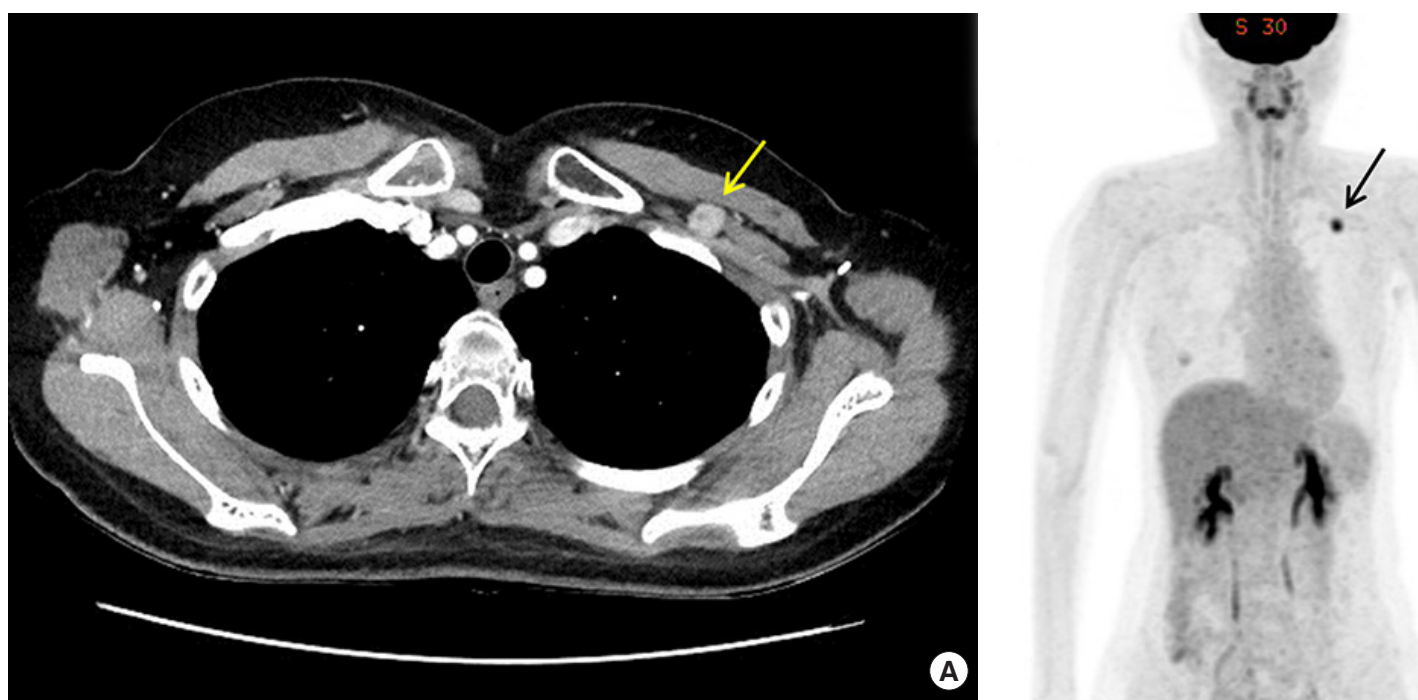

(B)
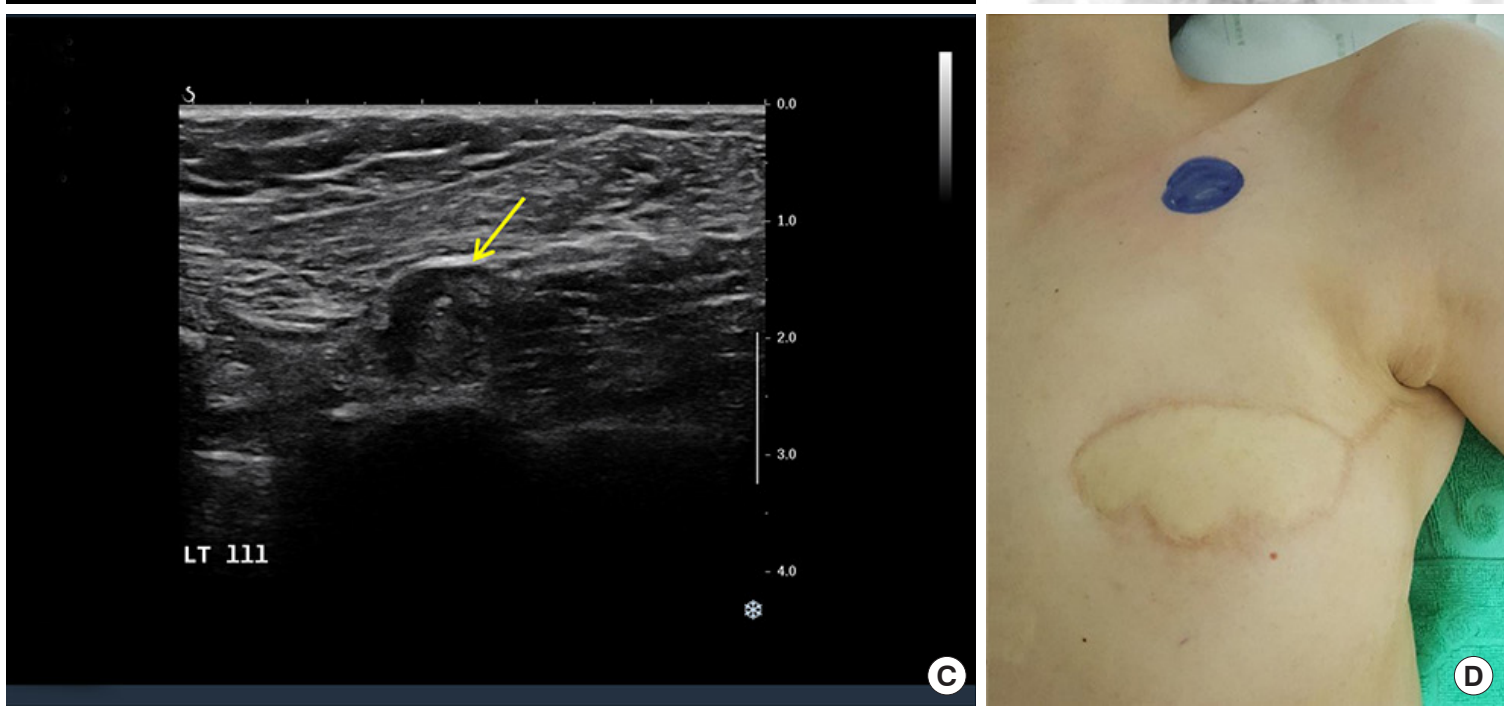

Figure 1. A 39-year-old female patient with breast cancer who underwent total mastectomy with breast reconstruction. (A) At 39 months after treatment, an enlarged infraclavicular lymph node (arrow) was detected on the chest CT scan. (B) The lymph node (arrow) showed the high uptake of 18F-FDG in PET/CT, indicating the recurrence of breast cancer. (C, D) Before surgery, the location of the recurrent lymph node (arrow) was evaluated with ultrasonography, and the location of the lymph node was marked on the skin. 
Surgical technique for isolated recurrent level III lymph nodes

Before surgery, the skin was marked by a radiologist, and preoperative tattooing with $1-3 \mathrm{~mL}$ of Charcotrace ${ }^{\mathrm{TM}}$ black ink (Phebra, New South Wales, Australia) was performed on the soft tissue around the recurrent lymph node (Figure 1D) [4].

The patients were placed in the supine position under general anesthesia with the ipsilateral arm abducted. The surgical team was composed of one breast surgeon and one vascular surgeon.

A transverse incision was made over the skin marking, and the pectoralis major muscle was split along its transverse fibers by electrocautery according to the tattooed path. The location of the recurrent lymph node was confirmed and identified by tactile sense. After the outline of the recurrent lymph node was identified to some extent, a tagging suture was made on the overlying fatty tissue. With traction of the suture, the recurrent lymph node was removed with the surrounding fatty tissue using multiple ties. In addition, the feeding vessel was completely tied up with black silk 3-0 and divided. After the removal of the recurrent lymph node, the surgical field was washed with $500 \mathrm{~mL}$ of normal saline, and bleeding control was performed. The drainage tube was placed on the excision site, and skin closure was performed with absorbable suture materials (Figure 2).

\section{RESULTS}

A total of 1,390 consecutive patients with operable breast cancer were followed up at Kyungpook National University Hospital with various laboratory tests and imaging studies for surveillance. The mean age was 49.82 (standard deviation $[\mathrm{SD}] \pm 9.90$ ) years, and the mean clinical and pathologic tumor size of the primary tumor was
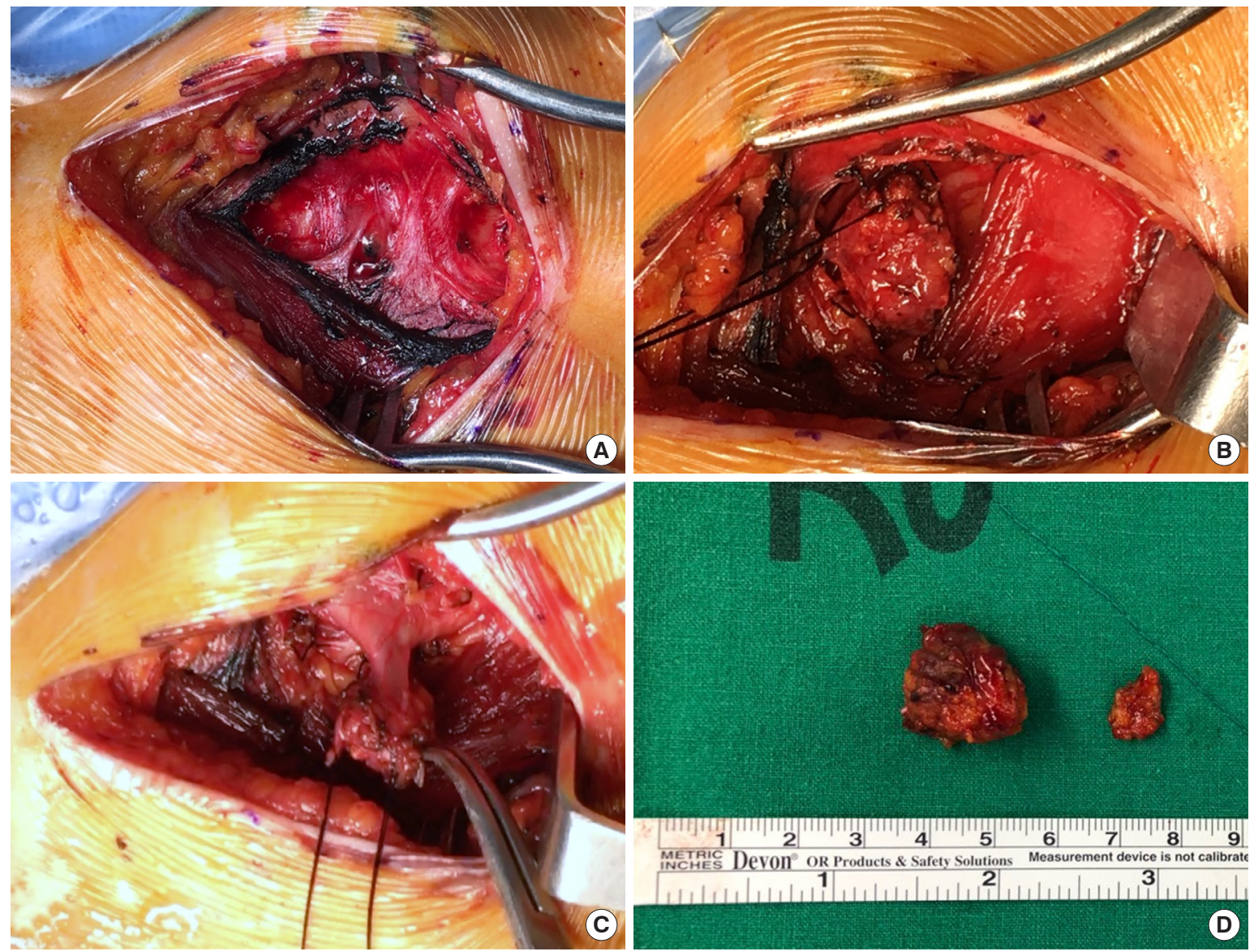

Figure 2. A surgical process for isolated level III lymph node recurrence in breast cancer. The photographs show the (A) split pectoralis major muscle along the tattooed path, (B) level III recurrent lymph node with tagging suture, (C) draining vein from the recurrent lymph node to the subclavian vein, and (D) resected specimen. 
$2.37(\mathrm{SD}, \pm 1.68)$ and $1.90(\mathrm{SD}, \pm 1.34) \mathrm{cm}$, respectively.

Most of the cases were those of invasive ductal carcinoma $(\mathrm{n}=1,062,76.41 \%)$. More than $60 \%$ of the patients had hormone receptor-positive breast cancer, and 105 patients (7.56\%) had triple-negative breast cancer. During the $94.81(\mathrm{SD}, \pm 193.42)$ months of the mean follow-up period, there were 85 cases (6.12\%) of locoregional recurrence, 95 cases (6.84\%) of distant metastasis, and 60 cases (4.32\%) of death.
Among the 85 cases of locoregional recurrence, isolated recurrent lymph nodes were observed in 25 cases. Three patients with an isolated recurrent infraclavicular lymph node received radiotherapy, and six patients underwent excision biopsy due to a history of radiotherapy for primary tumors. Additional treatment included chemotherapy or hormone treatment based on the biological characteristics of the recurred lesion (Figure 3).

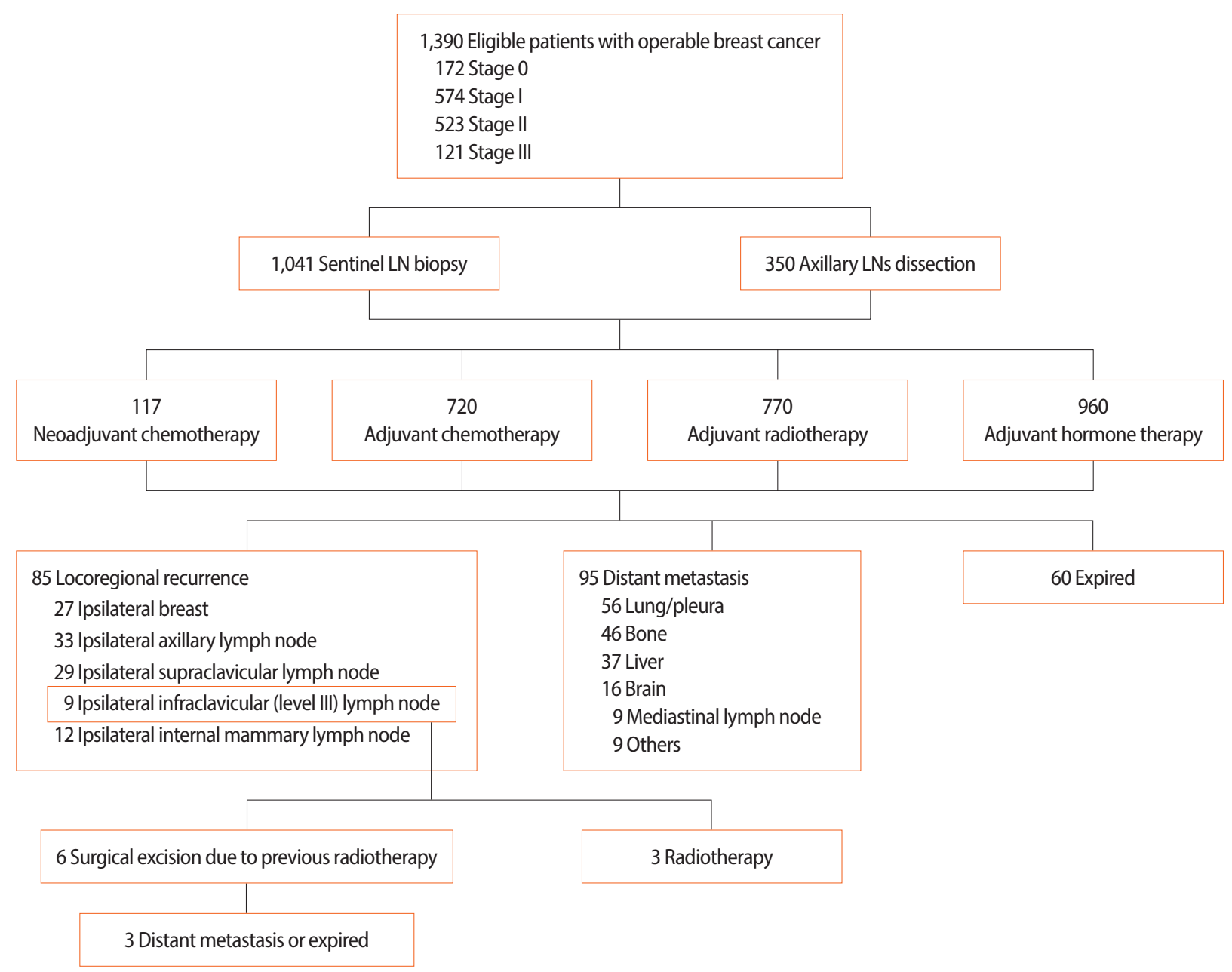

Figure 3. A flowchart showing management of breast cancer and oncologic outcomes.

Table 1. Clinical factors of patients with isolated level III recurrent axillary lymph node, which was removed by excision biopsy

\begin{tabular}{lclccc}
\hline No. & Age $(\mathrm{yr})$ & Initial tumor type & Disease-free interval (mo) & Additional oncologic event & Relapse-free interval (mo) \\
\hline 1 & 53 & Invasive ductal carcinoma & 200.10 & Death & 62.3 \\
2 & 70 & Invasive ductal carcinoma & 209.57 & - & - \\
3 & 59 & Ductal carcinoma in situ & 27.80 & - & - \\
4 & 42 & Invasive ductal carcinoma & 12.20 & Death & 45.6 \\
5 & 52 & Invasive ductal carcinoma & 31.43 & Distant metastasis & 12.7 \\
6 & 39 & Invasive ductal carcinoma & 37.13 & - & - \\
\hline
\end{tabular}


There was no failure in identifying the recurrent lymph node using the preoperative tattooing technique. The mean disease-free interval of the six patients with isolated level III recurrent lymph node was 86.37 (SD, \pm 92.18 ) months, and one patient was diagnosed with ductal carcinoma in situ. All of the six patients received radiotherapy as an initial treatment, and there was no intraoperative or postoperative major complication. Further oncological events occurred in three cases after surgery (distant metastasis $[\mathrm{n}=1]$; death $[\mathrm{n}=2])$ (Table 1 ).

\section{DISCUSSION}

Breast cancer is the most common form of cancer in women, and cancer-specific survival rates have increased $[1,5]$. However, prolonged survival can also lead to a higher chance of recurrence or metastasis [6].

The common metastatic organs of breast cancer are the bone, lungs, liver, and brain, and treatment may vary according to the metastatic lesion and tumor characteristics. Surgery for metastatic lesions is not strongly recommended as a treatment of choice [7]. However, locoregional recurrence can be treated with several local treatments including surgery or radiotherapy if possible, with additional advantages such as tumor burden reduction and the reevaluation of tumor characteristics [8]. These treatment strategies could provide useful information for systemic therapy [9-11].

Based on the NCCN guidelines, radiation therapy is prefuerred for the treatment of supraclavicular recurrence [12]. However, there is no standard treatment for infraclavicular level III lymph node recurrence. Nevertheless, as the infraclavicular area might have already been included in the radiotherapy boundary, surgical excision may be effective.

Anatomically, the infraclavicular lymph nodes are only visible when the pectoralis muscle is divided or transected. In such cases, preoperative ultrasound-guided localization with charcoal or blue dye for tattooing would be helpful to localize the lymph node. When the dye is injected, the surgeon can trace the tattooed path and easily identify the exact lymph node. As there could be tissue fibrosis due to previous radiotherapy in some cases, the vascular structure should be confirmed before surgery or surgery should be performed by a vascular surgeon.

In this study, patients with breast cancer having isolated level III lymph node recurrence were evaluated, and the findings demonstrated that recurrent lesions could be safely removed without failure of identification or long-term complications.

Isolated level III recurrent lymph nodes in breast cancer could be safely removed without major complications, even if the patients had received radiotherapy on the recurred site. This strategy would help improve the therapeutic effect of systemic treatment.

\section{CONFLICT OF INTEREST}

The authors declare that they have no competing interests.

\section{REFERENCES}

1. Park EH, Min SY, Kim Z, Yoon CS, Jung KW, Nam SJ, et al. Basic facts of breast cancer in Korea in 2014: the 10-year overall survival progress. J Breast Cancer 2017;20:1-11.

2. Yoshimura A, Ito H, Nishino Y, Hattori M, Matsuda T, Miyashiro I, et al. Recent improvement in the long-term survival of breast cancer patients by age and stage in Japan. J Epidemiol 2018;28:420-7.

3. Joshi S, Noronha J, Hawaldar R, Kundgulwar G, Vanmali V, Parmar V, Nair N, et al. Merits of level III axillary dissection in node-positive breast cancer: a prospective, single-institution study from India. J Glob Oncol 2019;5:1-8.

4. Kim WH, Kim HJ, Jung JH, Park HY, Lee J, Kim WW, et al. Ultrasound-guided restaging and localization of axillary lymph nodes after neoadjuvant chemotherapy for guidance of axillary surgery in breast cancer patients: experience with activated charcoal. Ann Surg Oncol 2018;25:494-500.

5. Wong FY, Tham WY, Nei WL, Lim C, Miao H. Age exerts a continuous effect in the outcomes of Asian breast cancer patients treated with breast-conserving therapy. Cancer Commun 2018;38:39.

6. Gerber B, Freund M, Reimer T. Recurrent breast cancer: treatment strategies for maintaining and prolonging good quality of life. Dtsch Arztebl Int 2010;107:85-91.

7. Redig AJ, McAllister SS. Breast cancer as a systemic disease: a view of metastasis. J Intern Med 2013;274:113-26.

8. Wapnir IL, Khan A. Current strategies for the management of locoregional breast cancer recurrence. Oncology 2019;33:19-25.

9. Friedel G, Linder A, Toomes H. The Significance of prognostic fac- 
tors for the resection of pulmonary metastases of breast cancer. Thorac Cardiovasc Surg 1994;42:71-5.

10. Friedel G, Pastorino U, Ginsberg RJ, Goldstraw P, Johnston M, Pass $\mathrm{H}$, et al, on behalf of the International Registry of Lung Metastases L, England. Results of lung metastasectomy from breast cancer: prognostic criteria on the basis of 467 cases of the international registry of lung metastases. Eur J Cardiothoracic Surg 2002;22:335-44.
11. Planchard D, Soria J-C, Michiels S, Grunenwald D, Validire P, Caliandro R, et al. Uncertain benefit from surgery in patients with lung metastases from breast carcinoma. Cancer 2004;100:28-35.

12. Goetz MP, Gradishar WJ, Anderson BO, Abraham J, Aft R, Allison KH, et al. NCCN guidelines insights: breast cancer, version 3.2018. J Natl Compr Canc Netw 2019;17:118-26. 